\title{
In vivo delivery of MMP3-shRNA and Sox9 lentivirus cocktail enhances matrix synthesis to prevent lumbar disc degeneration
}

\author{
Zheng Zhao $0^{1, A-F}$, Siyuan $L^{2, A-A, D, F}$, Hui Huang ${ }^{1, A, B, F}$, Jing Fang ${ }^{1, B, C, F}$, Huawei Wei ${ }^{1, B, C, F}$, Yongming Xi, ${ }^{1, A-F}$ \\ ${ }^{1}$ Department of Orthopedics, Affiliated Hospital of Qingdao University, China \\ ${ }^{2}$ Department of Orthopedics, Shandong Provincial Third Hospital, Jinan, China \\ A - research concept and design; B - collection and/or assembly of data; C - data analysis and interpretation; \\ $D$ - writing the article; $E$ - critical revision of the article; $F$ - final approval of the article
}

\section{Address for correspondence \\ Yongming Xi}

E-mail:yongming_xi@yahoo.com

\section{Funding sources}

The National Natural Science Foundation of China (NSFC, grant No. 81470104).

\section{Conflict of interest}

None declared

Received on September 22, 2019

Reviewed on March 31, 2020

Accepted on April 29, 2020

Published online on June 26, 2020

Cite as

Zhao Z, Li S, Huang H, Fang J, Wei H, Xi Y. In vivo delivery

of MMP3-shRNA and Sox9 lentivirus cocktail enhances matrix

synthesis to prevent lumbar disc degeneration. Adv Clin

Exp Med. 2020;29(6):639-647. doi:10.17219/acem/121509

DOI

10.17219/acem/121509

Copyright

Copyright by Author(s)

This is an article distributed under the terms of the

Creative Commons Attribution 3.0 Unported (CC BY 3.0)

(https://creativecommons.org/licenses/by/3.0/)

\begin{abstract}
Background. Intervertebral disc degeneration (IDD) is characterized by increased proteolytic degradation of the extracellular matrix (ECM), leading to a loss of collagen II and proteoglycan in the nucleus pulposus (NP). Although MMP3 has been reported to play a central role in disc degeneration, it is still unknown whether gene therapy targeting MMP3 can inhibit IDD.

Objectives. To investigate whether lentivirus-mediated MMP3 knockdown is capable of attenuating IDD. More importantly, we also explored whether combined gene therapy that simultaneously antagonizes MMP3 and overexpresses Sox9 can synergistically inhibit IDD and induce augmented matrix reconstitution in the degenerative NP.
\end{abstract}

Material and methods. We performed direct injection of lentiviral vectors LV-MMP3-shRNA and/or LVSox9 into rabbit lumbar discs. The animals were scanned using magnetic resonance imaging (MRI) at 8 , 12 and 24 weeks after the operation. We also evaluated the gene expression and synthesis of NP matrix components, including collagen II, aggrecan and proteoglycan.

Results. The MRI scans showed remarkable needle-puncture-induced progressive IDD in animals injected with PBS or $10 \wedge 7$ viral particles (VP) of the control virus. In contrast, injection of 10^7 VP of LV-MMP3-shRNA or LV-Sox9 substantially inhibited IDD. MMP3 knockdown or Sox9 overexpression stimulated collagen II and aggrecan expression, as well as proteoglycan synthesis. Notably, the injection of a cocktail of LV-MMP3-shRNA and LV-Sox9 $(5 \times 10 \wedge 6$ VP each) greatly delayed the development of IDD and induced the highest levels of collagen II and proteoglycan production, indicating a synergistic effect in ECM induction.

Conclusions. Our results suggest that gene therapy targeting MMP3 is an efficient way to delay IDD. Combined gene therapy possesses a stronger capacity to induce matrix components in degenerative NP tissue than single-gene delivery.

Key words: gene therapy, disc degeneration, SOX9, MMP3 


\section{Introduction}

Intervertebral disc degeneration (IDD) is a major cause of most musculoskeletal disorders of the spine that results in lower back pain, morbidity and physical disability. ${ }^{1}$ Although the precise etiology and pathophysiology of disc degeneration has not been fully elucidated, IDD is generally believed to be a consequence of increased proteolytic degradation of extracellular matrix (ECM) macromolecules. During the disc degenerative process, the nucleus pulposus (NP) loses water and large amounts of aggregating proteoglycans and type II collagen (COL2A1), which leads to ECM breakdown and structural failure. ${ }^{2}$

A variety of studies have focused on the mechanisms underlying ECM degradation in IDD pathogenesis. An increasing amount of evidence has shown that matrix metalloproteinases (MMPs) play important roles in the degradation of matrix components during the disc degenerative process. Several MMP family members, such as MMP1, MMP2, MMP3, MMP7, MMP8, MMP9, MMP12, and MMP26, have been reported to be increasingly expressed in degenerated disc tissues, suggesting an association between MMP and IDD. ${ }^{3-6}$ Notably, Bachmeier et al. reported that among all MMPs tested (MMP1, MMP2, MMP3, MMP7, MMP8, MMP9, and MMP13), the expression of MMP3 was consistently and substantially upregulated in degenerated disc samples, and this process was accompanied with increased enzymatic activity of MMP3, ${ }^{7}$ implying that MMP3 plays a central role in disc degeneration. Besides elevated matrix-degrading MMPs, the loss of matrix-producing Sox9 also plays a role in ECM degradation in IDD. Being a key transcription factor in chondrogenic differentiation, Sox 9 can directly stimulate gene expression of collagen type II and aggrecan, ${ }^{8}$ the major core protein in proteoglycan. Sive et al. detected a strong signal of Sox9 in normal NP tissues using in situ hybridization, which was accompanied by high-level expression of collagen II and aggrecan. ${ }^{9}$ However, the expression of Sox 9 decreases with aging and disc degeneration. ${ }^{10}$ These studies suggest that MMP-induced excessive ECM degradation or loss of Sox9-reduced ECM synthesis is critically implicated in the pathogenesis of IDD. Hence, molecular therapy which targets MMP or restores Sox 9 can be used to reconstitute matrix components in a degenerative NP.

Although spine disorders resulting from disc degeneration can be treated with surgery, including discectomy or spinal fusion, they are not curative and are associated with various complications. Non-surgical treatments - such as drugs, massage or physical therapy - however, can only relieve the clinical symptoms; they cannot delay or reverse the disc degenerative process. As a result, gene therapy has received considerable interest, as this approach can transfer the genes of therapeutic proteins to the disc cells, enabling them to manufacture the proteins endogenously, on a continuous or regular basis, allowing for long-term regulation of matrix synthesis with the potential to prevent or delay IDD. ${ }^{11}$ Nishida et al. conducted an initial study showing that direct injection of adenoviral TGF $\beta 1$ vectors into the rabbit lumbar discs led to a $100 \%$ increase in proteoglycan synthesis compared with intact control tissues. ${ }^{12}$ Since then, gene therapy using several growth factors, including BMP2, GDF5, IGF1, and interleukin (IL)-1 receptor antagonist (IL-1Ra) for disc degeneration has been tested both in vitro and in vivo, as previously reported. ${ }^{11,13}$ Co-transduction of adeno-associated virus (AAV)-based Sox 9 and OP1 vectors was reported to exhibit a stronger effect in the induction of proteoglycan and collagen II than AAV-Sox 9 or AAV-OP1 alone. ${ }^{14}$

So far, gene therapy which targets an individual MMP molecule (particularly MMP3) against disc degeneration has not yet been reported. In our study, we performed direct injection of lentiviral vectors expressing MMP3-shRNA into NP tissues of lumbar discs in rabbits and evaluated the reconstitution of matrix components, specifically collagen type II and proteoglycan. We also investigated whether a cocktail of both MMP3-shRNA and Sox9 lentiviruses could trigger a synergistic effect to further augment matrix synthesis.

\section{Material and methods}

\section{Lentiviral vectors}

The lentiviral vectors harboring MMP3-shRNA (LVMMP3-shRNA), Sox9 (LV-Sox9), or empty vectors were provided by Biowit Technologies (Shenzhen, China). All lentivirus vectors were stored at $-80^{\circ} \mathrm{C}$ and diluted with phosphate-buffered saline (PBS) to a concentration of $5 \times 10^{5}$ viral particles $(\mathrm{vp}) / \mu \mathrm{L}$ right before use.

\section{Animal study}

This is an experimental study in a rabbit model. The protocol for the animal study was reviewed and approved by the Animal Care Committee of Qingdao University, China. A total of $25 \mathrm{New}$ Zealand white rabbits were used in this study, with an average age of 4 months and an average body weight of $2.5 \mathrm{~kg}$. Before surgery, the animals underwent magnetic resonance imaging (MRI) to exclude congenital spine deformity and disc disease.

For direct gene transfer, the animals were anesthetized with an intramuscular injection of $1.5 \%$ pentobarbital sodium at a dosage of $2 \mathrm{~mL} / \mathrm{kg}$. Following anesthesia, each animal was placed in a lateral position with all 4 limbs fixed. A vertical incision of about $3 \mathrm{~cm}$ was made from the iliac crest to the lower edge of the $12^{\text {th }}$ rib via a right extraperitoneal approach. The peritoneum was bluntly dissected to expose the transverse processes from L3 to L7. A 24-gauge needle was used to pierce into the center of the exposed L3/4, L4/5, L5/6, and L6/7 lumbar discs ( $~ 5 \mathrm{~mm})$ parallel to the upper and lower endplates. Then, a volume of $20 \mu \mathrm{L}$ 


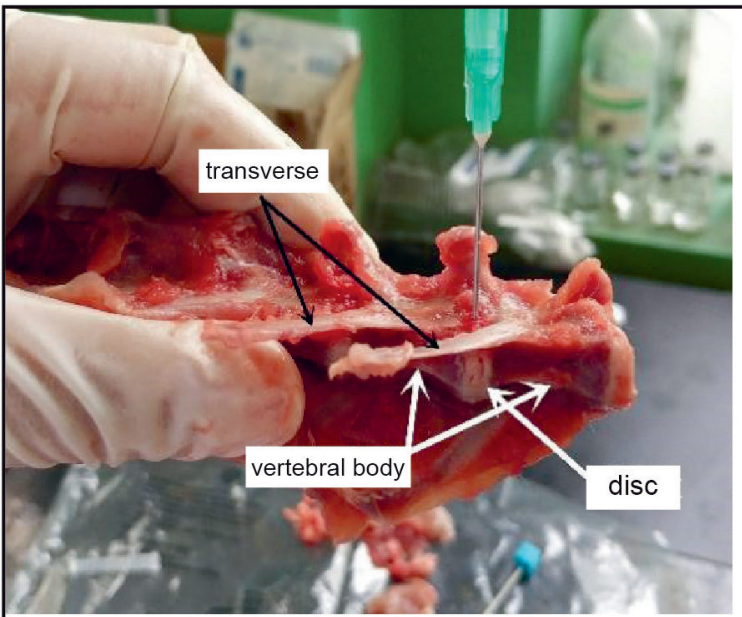

Simulated needle puncture into the disc

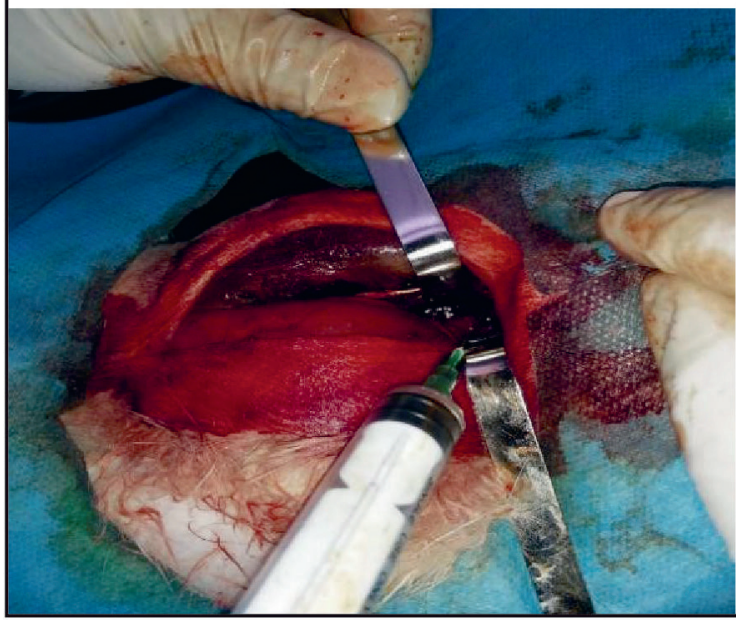

Injection into the disc

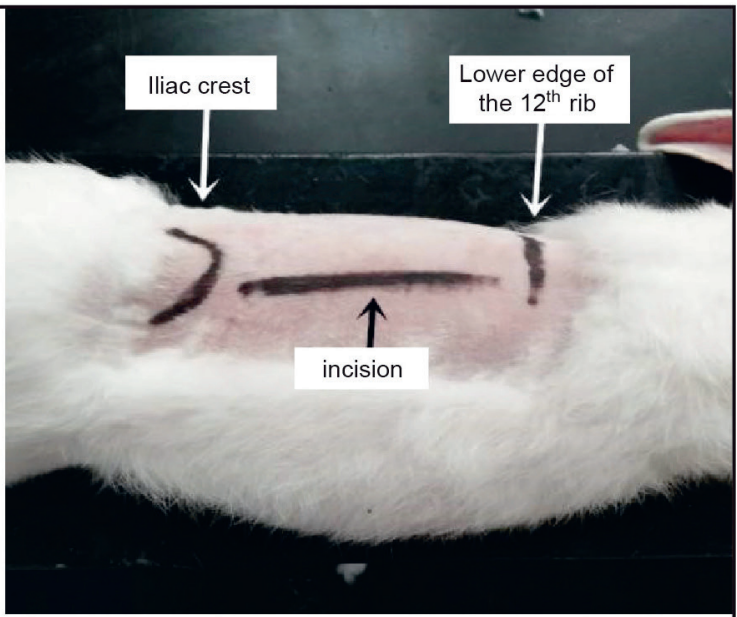

Surgical approach

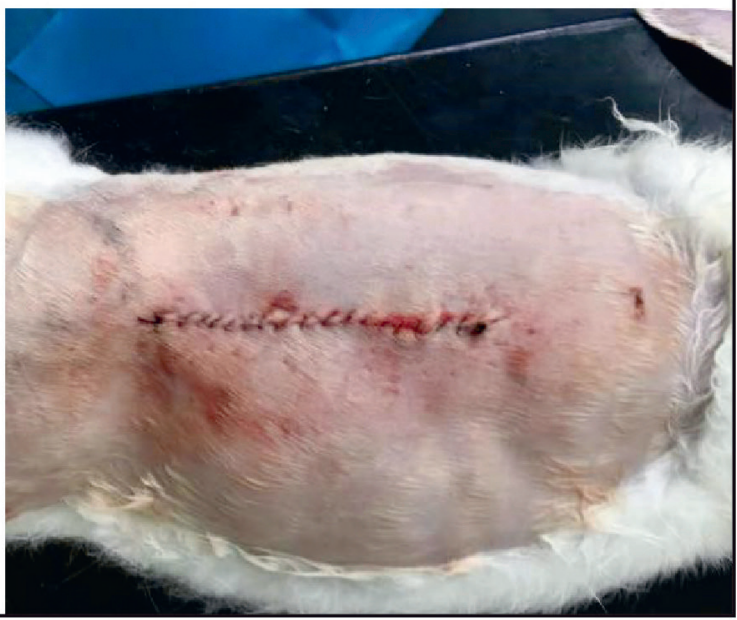

Incision suture

Fig. 1. In vivo gene delivery. Lentiviral vectors are directly injected into the rabbit lumbar discs $(\mathrm{L} 3 / 4, \mathrm{~L} 4 / 5, \mathrm{~L} 5 / 6$, and L6/7) through a surgical approach

containing PBS or $10^{7}$ vp of lentivirus was injected directly into the NP tissue. All 25 rabbits were randomly divided into 5 groups and injected with a total volume of $20 \mu \mathrm{L}$ of PBS or lentivirus, as follows: group I - PBS; group II $-10^{7} \mathrm{vp}$ of empty virus (control); group III - $10^{7} \mathrm{vp}$ of LV-MMP3shRNA; group IV $-10^{7} \mathrm{vp}$ of Lento-Sox9; and group V (cocktail) $-5 \times 10^{6} \mathrm{vp}$ of LV-MMP3-shRNA $+5 \times 10^{6} \mathrm{vp}$ of LV-Sox9. After injection, the opening was closed with sutures (Fig. 1). The rabbits were administered penicillin (800,000 units) intramuscularly before and after the operation. No incidences of infection or death were reported.

\section{MRI assessment}

The animals were examined with MRI at 8, 12 and 24 weeks after the operation. A Siemens Avanto 3.0T medical superconductive MRI scanner (Siemens AG, Munich, Germany) was used to scan the sagittal lumbar spine of the animals with a cervical coil. The parameters of the scanner were as follows: T2-weighted sequence repetition time - 2,000-6,000 ms; echo time - 80-120 ms; bandwidth $-25 \mathrm{~Hz}$; microwave -16 ; matrix $-384 \times 224 \mathrm{px}$; nex -4 ; field of vision $-16 \times 16 \mathrm{~cm}$; thickness $-3 \mathrm{~mm}$; and spacing $-0.2 \mathrm{~mm}$. A degenerative disc mainly emitted a low signal, that is, the T2-weighted signal intensity decreased and the disc became thinner. Based on the changes in the T2-weighted signal, the improved Thompson classification method was used as the standard. The MRI data was blindly assessed by 2 independent senior radiologists.

\section{Quantitative real-time polymerase chain reaction}

At 24 weeks post-operation, the animals were sacrificed and the NP tissues were harvested. Total RNA was isolated using TRIzol reagent (Amresco, Solon, USA) and was reverse-transcribed to cDNA using qScript cDNA SuperMix (Quanta Biosciences, Woodbridge, 
USA). Quantitative real-time polymerase chain reaction (qRT-PCR) was conducted with SYBR green master mix. The primers for rabbit genes were listed as follows: $\mathrm{CO}$ L2A1, forward 5'-GGATAGA CCCCAACCAAGGC-3', reverse 5'-GCTGCTCCACCAGTTCTTCT-3'; MMP3, forward 5'-AGCCAATGGAAATGAAAACTCTTC-3', reverse 5'-CCAGTGGATAGGCTGAGCAAA-3'; Sox9, 5'-GGTGCTCAAGGGCTACGACT-3', reverse 5'-GGGTGGTCTTTCTTGTGCT G-3'; Actin, forward 5'-CTGGAACGGTGAAGGTGACA-3', reverse 5'-CGGCCACATTGC AGAACT-3'. The PCR products were analyzed with an ABI PRISM 7900HT Sequence Detection System (Applied Biosystems, Foster City, USA). The cycling conditions were $50^{\circ} \mathrm{C}$ for $2 \mathrm{~min}$ and $95^{\circ} \mathrm{C}$ for $10 \mathrm{~min}$, followed by 40 repetitions of $95^{\circ} \mathrm{C}$ for $15 \mathrm{~s}$ and $60^{\circ} \mathrm{C}$ for $1 \mathrm{~min}$. One dissociation stage $\left(95^{\circ} \mathrm{C}\right.$ for $15 \mathrm{~s}, 60^{\circ} \mathrm{C}$ for $15 \mathrm{~s}$ and $95^{\circ} \mathrm{C}$ for $15 \mathrm{~s}$ ) was added to produce the melting curve at the end of the cycling condition. Relative mRNA concentrations of the target genes were determined with ABI software (RQ Manager v. 1.2; Thermo Fisher Scientific, Waltham, USA), which normalizes the target gene threshold cycle to that of endogenous GAPDH transcripts $(\Delta \Delta \mathrm{Ct})$, using the formula $2^{-\Delta \Delta C t}$ to determine fold change.

\section{Western blot}

For western blot, cell lysates were isolated from NP tissues with RIPA lysis buffer (10 mM of Tris, pH 7.4, $100 \mathrm{mM}$ of $\mathrm{NaCl}, 1 \mathrm{mM}$ of EDTA, $1 \mathrm{mM}$ of EGTA, $1 \mathrm{mM}$ of $\mathrm{NaF}$, $20 \mathrm{mM}$ of $\mathrm{Na}_{4} \mathrm{P}_{2} \mathrm{O}_{7}, 2 \mathrm{mM}$ of $\mathrm{Na}_{3} \mathrm{VO}_{4}, 1 \%$ Triton X-100, $10 \%$ glycerol, $0.1 \%$ sodium dodecylsulphate (SDS), and $0.5 \%$ deoxycholate). Protein concentrations were estimated using Bradford protein assay (Bio-Rad, Hercules, USA). An equal amount of protein $(40 \mu \mathrm{g})$ was resolved using 10\% SDS-PAGE and transferred to polyvinylidene difluoride (PVDF) membrane (Thermo Fisher Scientific). Non-specific binding sites were blocked with 5\% nonfat dry milk (Roche, Basel, Switzerland) in Tris-buffered saline containing $0.1 \%$ Tween (TBST). The membrane was incubated with primary antibody against rabbit COL2A1 or aggrecan (Abcam, Cambridge, UK) in TBST at $4^{\circ} \mathrm{C}$ overnight on a shaker, and was then incubated with horseradish peroxidase (HRP)-conjugated secondary antibodies for $1 \mathrm{~h}$ at room temperature. The immunoblots were visualized using Western ECL Blotting Substrates (Bio-Rad). The protein bands were analyzed for densitometry using ImageJ software (National Institutes of Health, Bethesda, USA).

\section{Proteoglycan assay}

The amount of proteoglycan in the NP tissues was examined through determining soluble sulfated glycosaminoglycans (sGAG) using a Proteoglycan Detection Kit (Amsbio, Cambridge, USA) according to the manufacturer's protocol. Briefly, NP tissue was minced, homogenized and incubated with $1 \mathrm{~mL}$ of $20 \mathrm{mM}$ sodium phosphate buffer
(pH 6.8) containing $1 \mathrm{mM}$ of EDTA, $2 \mathrm{mM}$ of dithiothreitol and $300 \mu \mathrm{g}$ of papain at $60^{\circ} \mathrm{C}$ for $4 \mathrm{~h}$ until the tissue was soluble. Iodoacetic acid was added to a final concentration of $10 \mathrm{mM}$ and was mixed well with $1 \mathrm{~mL} 50 \mathrm{mM}$ Tris/ $\mathrm{HCl}$ (pH 8.0). Both the samples and the sGAG standards were mixed with DMB dye. Absorbance was read in a microplate reader at an optical density (OD) of $520 \mathrm{~nm}$.

\section{Statistical analyses}

The data was reported as means \pm standard deviation (SD) (or means \pm standard error of the mean (SEM); see figure legends) of at least 3 independent trials, and was analyzed with a two-tailed Student's t-test for 2 groups, or one-way analysis of variance (ANOVA) for multiple groups using GraphPad Prism software (GraphPad Software Inc, San Diego, USA). The variance is similar between the groups in the same trial. A p-value $<0.05$ was considered statistically significant.

\section{Results}

\section{MRI reveals delayed disc degeneration by LV-MMP3-shRNA or LV-Sox9 vectors}

Previous studies have reported that puncturing with a needle can induce progressive disc degeneration in rabbits. ${ }^{15-17}$ In our study, we performed direct injection of PBS, empty lentivirus, and lentivirus expressing MMP3-shRNA and/or Sox9 into rabbit lumbar discs, to explore whether gene therapy-mediated MMP3 knockdown or Sox 9 overexpression could delay the development of IDD. The preoperative T2-weighted MRI scans revealed strong and uniform signals for each lumbar disc (Fig. 2A). However, at 8 weeks post-injection, the T2-weighted signal intensity decreased in the discs injected with PBS or empty virus, indicating an early degenerative process. Interestingly, discs injected with LV-MMP3-shRNA, LV-Sox9 or combined LV-MMP3shRNA+LV-Sox9 vectors still appeared normal (Fig. 2B). At 12 weeks after the operation, MRI scans still displayed an intact structure in the PBS- or empty-virus-treated discs, although a more pronounced reduction of signal intensity was identified, indicating a progressively advanced NP degeneration. The MMP3-shRNA- or Sox9-lentivirusinjected discs also displayed a decrease in signal intensity, though to a much lesser extent. However, the cocktail injection of MMP3-shRNA and Sox9 lentivirus led to an only slightly reduced MRI signal (Fig. 2C). At 24 weeks after surgery, PBS or empty-virus-injected lumbar discs exhibited a strikingly less intense MRI signal with a loss of disc integrity, indicating severe disc degeneration. In contrast, the MMP3-shRNA- or Sox9-lentivirus-injected discs remained intact, even though the T2-weighted MRI scan showed reduced signal intensity. Notably, the discs injected with a cocktail of MMP3-shRNA and Sox9 lentiviruses not 
A

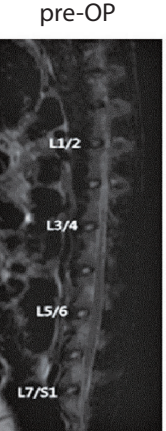

B

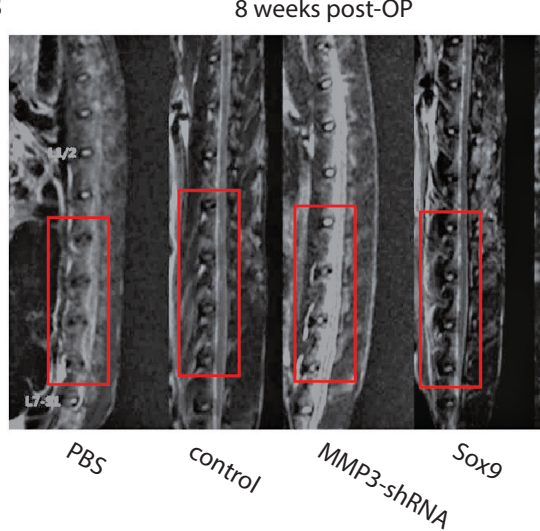

C
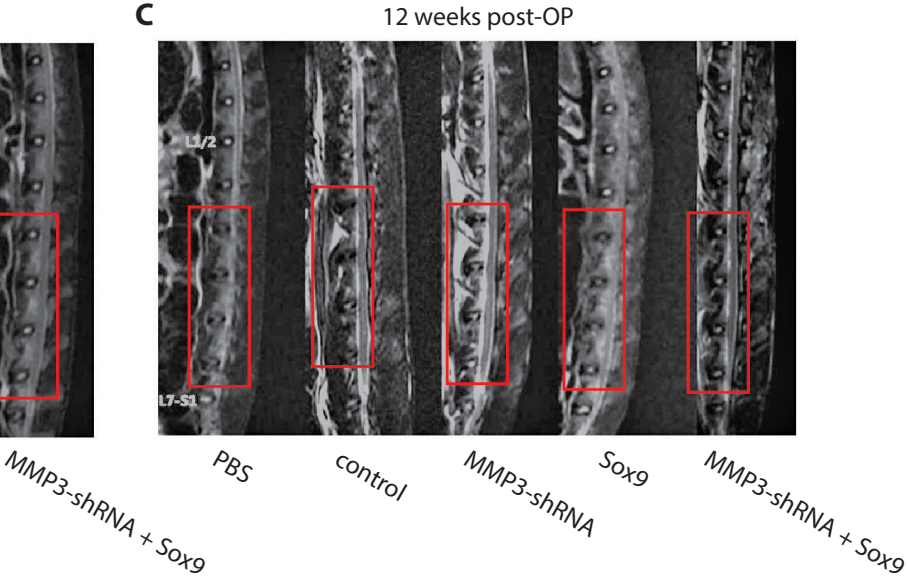

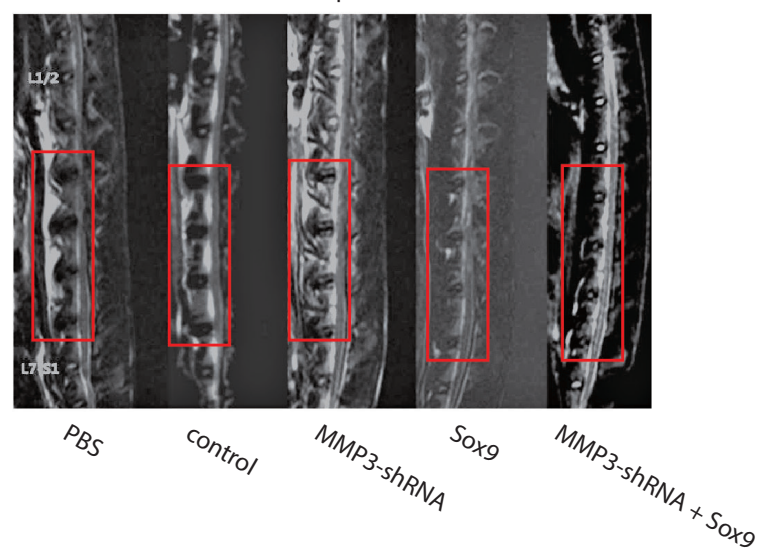

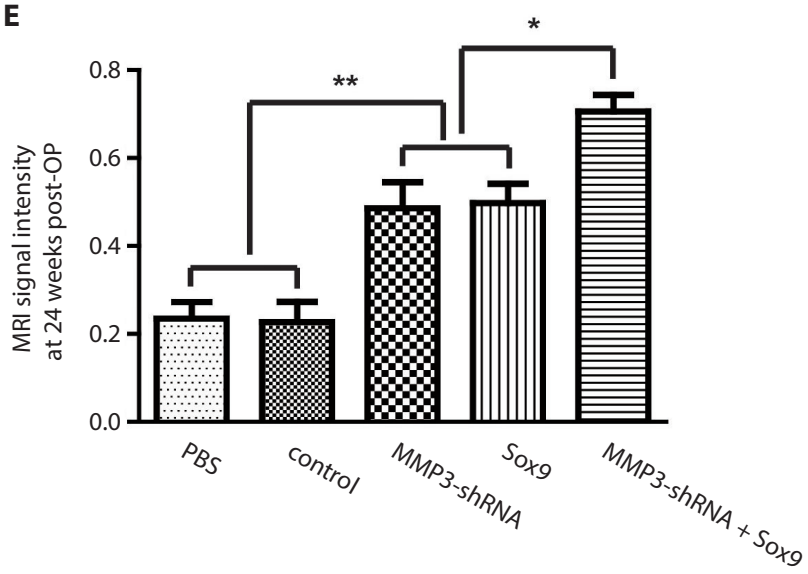

Fig. 2. MRI analysis. Lumbar discs are scanned using T2-weighted MRI at different time points: (A) before the operation (pre-OP), (B) 8 weeks after operation, (C) 12 weeks after the operation, and (D) 24 weeks after the operation. (E) MRI signal intensity at 24 weeks after the operation. Red boxes surround L3/4, $L 4 / 5, L 5 / 6$, and L6/7 intervertebral discs. Note that lentiviral delivery of MMP3-shRNA or Sox9 greatly delays needle-puncture-induced disc degeneration when compared to PBS or empty virus treatment, whereas combined gene delivery induces a synergistic effect in the attenuation of IDD

only appeared intact, but also displayed only a moderate decrease in MRI signal intensity (Fig. 2D,E; Table 1).

Based on the comparison of MRI signal intensity among different groups, lentivirus-mediated MMP3 knockdown or Sox 9 overexpression efficiently delayed the progression of needle-puncture-induced disc degeneration. Importantly, injection of combined LV-MMP3-shRNA and LV-Sox9 vectors significantly exerted a greater capacity than MMP3-shRNA ( $\mathrm{p}=0.026)$ or Sox $9(\mathrm{p}=0.017)$ single transgene to delay the development of IDD, indicating a synergistic effect of cocktail gene delivery.

\section{Lentiviral MMP3-shRNA or Sox9 gene transfer mediates long-term transgene expression}

The animals were sacrificed at 24 weeks after the operation. The NP tissues were harvested from their lumbar discs for RNA and protein assays. We first evaluated transgene expression using qRT-PCR analysis and found that, compared to the control group, the in vivo transfer of LV-MMP3-shRNA vectors resulted in a $65 \%$ decrease in MMP3 level. Interestingly, the injection of both lentiviruses decreased MMP3 mRNA to a level comparable to that of LV-MMP3-shRNA alone (Fig. 3A). On the other hand, LV-Sox9 viral vectors led to a 3.8-fold increase in Sox 9 mRNA in comparison with the controls. Similarly, the cocktail of lentiviruses induced a 3.5-fold increase of Sox9 mRNA (Fig. 3B). These results not only suggest that lentiviral vectors can mediate long-term in vivo gene expression, but they also imply that combined transduction of 2 transgenes can stimulate higher gene expression than single-gene delivery.

\section{Lentiviral MMP3-shRNA or Sox9 gene transfer increases collagen II expression}

Given that the loss of collagen II and proteoglycan are the predominant pathological changes in IDD, we first examined whether in vivo gene delivery of lentivirus vectors could restore the expression of collagen II in NP tissues during a needle-puncture-induced degenerative process. Using qRT-PCR analysis, we found that injecting LV-MMP3-shRNA and LV-Sox9 induced a 1.82- and 
A

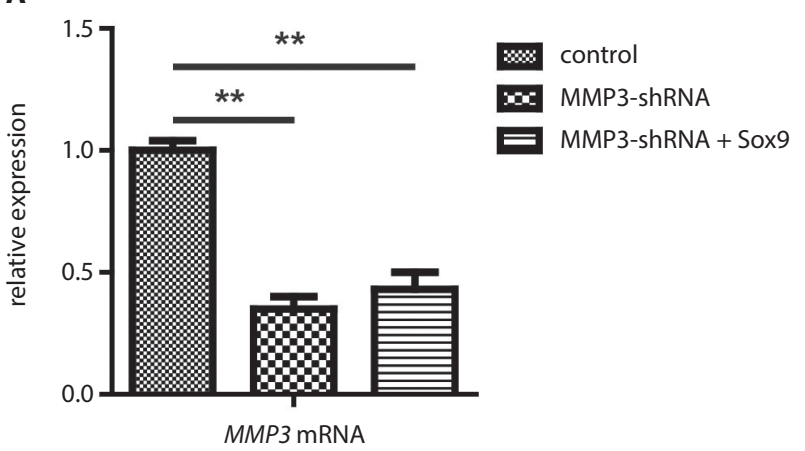

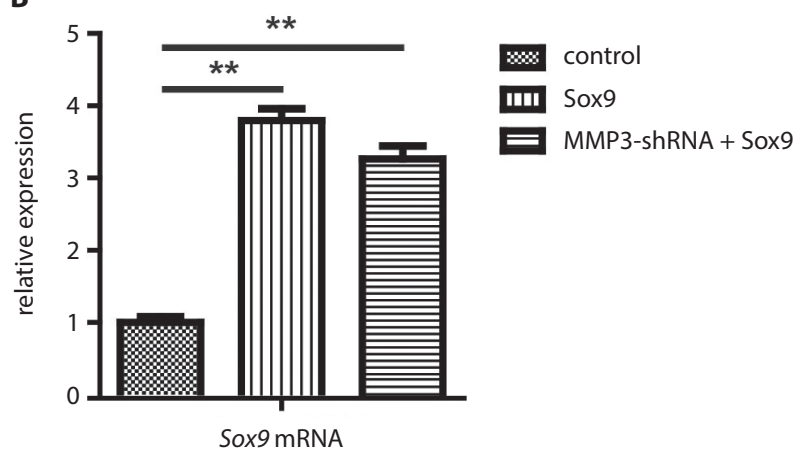

Fig. 3. Lentiviral gene delivery mediates long-term transgene expression. A. qRT-PCR analysis for MMP3 mRNA in NP tissue. B. qRT-PCR analysis for Sox9 mRNA in NP tissue. Data is presented as means \pm SEM. Note that cocktail gene delivery induces MMP3 or Sox9 mRNA to a comparable extent as the delivery of MMP3-shRNA or Sox9 lentivirus alone

** $p<0.01$

Table 1. MRI signal intensity (modified Thompson classification)

\begin{tabular}{|l|c|c|c|}
\hline \multicolumn{1}{|c|}{ Group } & Pre-OP & 8 weeks & 12 weeks \\
\hline PBS & $1.000 \pm 0.051$ & $0.677 \pm .097$ & $0.367 \pm 0.081$ \\
\hline Control & $1.000 \pm 0.103$ & $0.652 \pm 0.083$ & $0.383 \pm 0.043$ \\
\hline MMP3-shRNA & $1.000 \pm 0.072$ & $0.836 \pm 0.059$ & $0.235 \pm 0.037$ \\
\hline Sox9 & $1.000 \pm 0.067$ & $0.845 \pm 0.064$ & $0.227 \pm 0.046$ \\
\hline MMP3-shRNA+Sox9 & $1.000 \pm 0.086$ & $0.903 \pm 0.069$ & 0.077 \\
\hline
\end{tabular}

* Analysis between different groups as follows.

\begin{tabular}{|c|c|c|}
\hline Groups & Fold change & $p$-value \\
\hline MMP3-shRNA+Sox9 vs Sox9 & 1.42 & $<0.05$ \\
\hline MMP3-shRNA+Sox9 vs MMP3-shRNA & 1.45 & $<0.05$ \\
\hline MMP3-shRNA+Sox9 vs control & 3.11 & $<0.05$ \\
\hline MMP3-shRNA+Sox9 vs PBS & 3.00 & $<0.01$ \\
\hline Sox9 vs MMP3-shRNA & 1.02 & $>0.05$ \\
\hline Sox9 vs controls & 2.19 & $<0.05$ \\
\hline Sox9 vs PBS & 2.12 & $<0.05$ \\
\hline MMP3-shRNA vs controls & 2.14 & $<0.05$ \\
\hline MMP3-shRNA vs PBS & 2.07 & $<0.05$ \\
\hline Controls vs PBS & 0.09 & $>0.05$ \\
\hline
\end{tabular}

1.62-fold increase in Col $2 \alpha 1$ mRNA, respectively, compared to the controls. Notably, the combined injection (LV-MMP3-shRNA+LV-Sox9) induced the highest level of COL2A1 mRNA among all 5 groups, which led to a 2.3fold increase when compared with the controls, and a 1.26or 1.41-fold increase compared to the LV-MMP3-shRNAor LV-Sox9-treated group, respectively (Fig. 4A).

Using western blot analysis, we found that the transduction of LV-MMP3-shRNA or LV-Sox9 dramatically upregulated collagen II at the protein level: 3.11- and 3.33-fold compared to the controls. Similarly, injecting a MMP3shRNA and Sox9 lentivirus cocktail led to the highest induction of COL2A1 among all groups tested, which was a 3.88-fold increase in comparison with the controls (Fig. 4B). These results suggest that direct in vivo gene therapy using lentiviral MMP3-shRNA or Sox9 vectors promotes the expression of collagen II in degenerative NP tissues. Our findings also reveal that cocktail delivery of MMP3-shRNA and Sox9 lentiviruses can initiate a synergistic effect to augment collagen II expression.

\section{Lentiviral MMP3-shRNA or Sox9 gene transfer increases proteoglycan synthesis}

We then evaluated the production of proteoglycan, the primary ECM matrix in NP tissues, by assessing the expression of aggrecan (the major core protein in proteoglycan), as well as the synthesis of sGAG. Western blot analysis showed that lentivirus-mediated MMP3 knockdown increased aggrecan expression 2.3-fold compared to the controls. LV-Sox9, however, only slightly (but significantly $-\mathrm{p}=0.043$ ) stimulated aggrecan expression. 

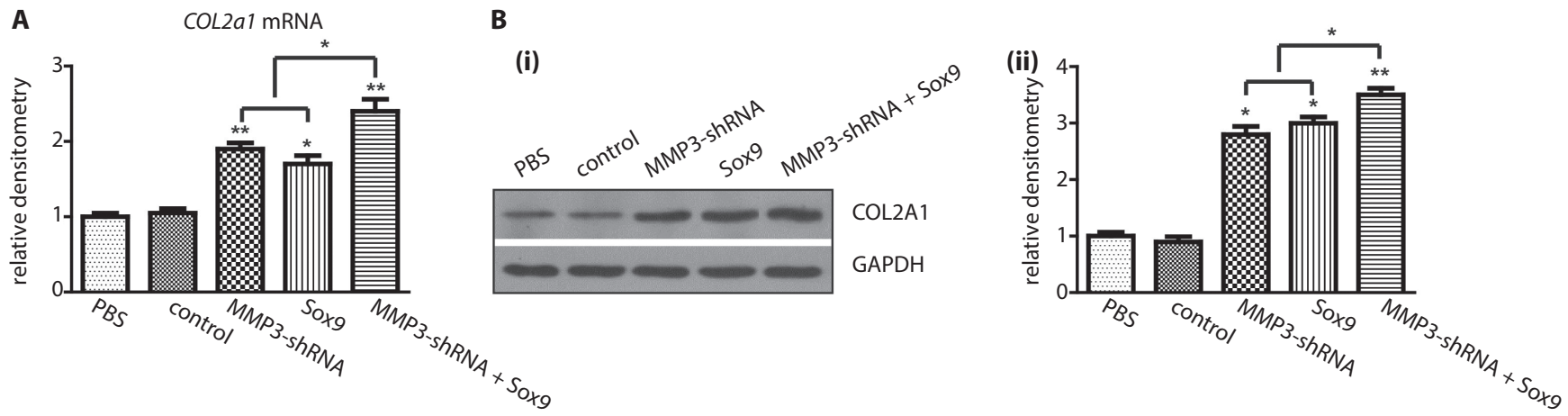

Fig. 4. MMP3 knockdown or Sox9 overexpression induces collagen Il expression. A. qPCR analysis for COL2A1 mRNA. B. Western blot analysis for COL2A1 (i) and densitometry of protein bands normalized with GAPDH using ImageJ (ii). The p-values on top of the bars denote the comparison between each lentiviral transgene group and an empty lentivirus group. For (A), data is presented as means \pm SEM. For (B), data is presented as mean \pm SD

${ }^{*} p<0.05 ;{ }^{* *} p<0.01$.

A

(i)

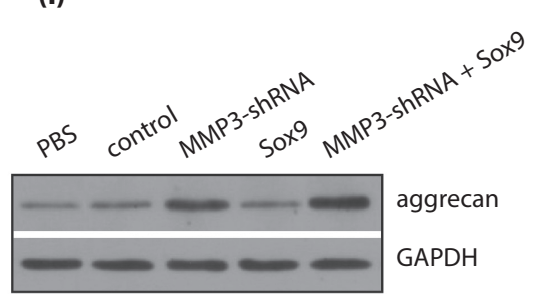

B

(ii)

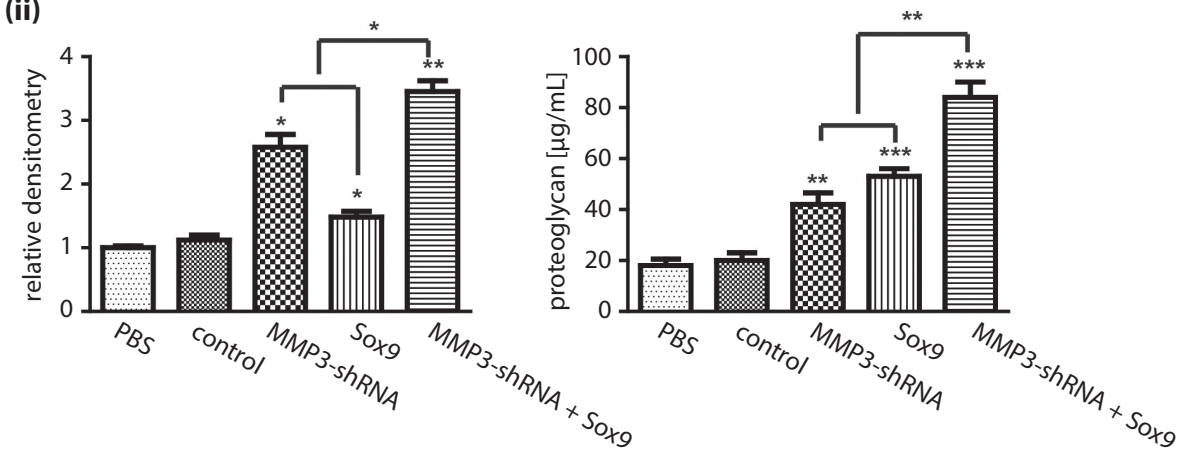

Fig. 5. MMP3 knockdown or Sox9 overexpression stimulates proteoglycan production. A. Western blot analysis for aggrecan (i) and densitometry of protein bands normalized with GAPDH using ImageJ (ii). B. Proteoglycan assay in NP tissue. The p-values on top of the bars denote the comparison between each lentiviral transgene group and an empty lentivirus group. For (A), data is presented as means $\pm S D$. For (B), data is presented as means $\pm S E M$

${ }^{*} p<0.05 ;{ }^{* *} p<0.01 ; * * * 00.001$.

In contrast, the injection of both lentiviruses resulted in the highest expression of aggrecan, which was a 3.08fold increase in comparison with the controls (Fig. 5A).

Besides the upregulation of aggrecan by lentivirus, we found that the knockdown of MMP3 or overexpression of Sox9 resulted in a 2.1- and 2.65-fold higher synthesis of sGAG, respectively, compared to the controls. Strikingly, injecting both LV-MMP3-shRNA and LV-Sox9 vectors led to the strongest induction of sGAG: a 4.2-fold increase (Fig. 5B). These results suggest that lentivirus-mediated MMP3 knockdown or Sox9 overexpression increases proteoglycan production, and more importantly, indicates that the injection of a MMP3-shRNA/Sox9 lentivirus cocktail triggers a synergistic effect to enhance proteoglycan synthesis.

\section{Discussion}

As the major cause of lower back pain, IDD is characterized by excessive degradation of the ECM, particularly the type II collagen and aggrecan in NP tissues. ${ }^{18}$ Since the matrix in NP mediates lumbar discs' resistance to compressive stresses, ${ }^{10}$ the reconstitution of matrix components using gene therapy is of great importance in the delay or attenuation of IDD. The family of MMPs is thought to constitute the major catabolic enzymes in the intervertebral disc. ${ }^{8}$ Increased MMP expression and/or activity have been implicated in the pathogenesis of IDD. Although gene therapy using viral or non-viral vectors to overexpress growth factors or cytokines has received considerable interest, a therapy that directly antagonizes MMP to prevent disc degeneration has not been studied, and this warrants investigation.

In our study, we established a needle-puncture-induced IDD model in rabbits, as reported previously. ${ }^{15-17}$ The MRI scans revealed that the lumbar discs injected with PBS or empty lentivirus underwent degenerative process as early as after 8 weeks, and the disc degeneration became extremely pronounced 24 weeks after injection. However, injecting lentivirus carrying MMP3-shRNA greatly delayed the progression of IDD on MRI, suggesting that the inhibition of MMP3 is effective in preventing disc degeneration. Biochemical assays further suggested 
that lentivirus-mediated MMP3 knockdown increased the expression of collagen II at both the mRNA and protein levels. This finding is interesting because MMP3 mainly degrades collagen II and other types of collagen (e.g., III, IV, IX, and X), but it cannot degrade collagen $I,{ }^{19}$ which is the predominant type of collagen fibers in the later stages of disc degeneration. As a result, the knockdown of MMP3 using gene therapy not only avoids degradation of collagen II in the NP, but it does so without affecting the degradation of collagen I by other MMPs. Additionally, lentiviral delivery of MMP3-shRNA increased the production of proteoglycan, as evidenced by the upregulated expression of aggrecan and the synthesis of sGAG. These findings suggest that gene therapy targeting MMP3 represents an efficient approach against IDD.

As a master regulator for chondrogenic differentiation, Sox9 directly drives collagen II and aggrecan gene expression. ${ }^{8}$ A loss of Sox 9 is closely associated with disc degeneration. ${ }^{10}$ In our study, direct in vivo gene delivery of the Sox9 lentivirus attenuated the progression of lumbar disc degeneration, as indicated by MRI scans. Overexpression of Sox9 also increased the production of collagen II and proteoglycan, suggesting that lentiviral Sox9 gene delivery can be used to alleviate IDD through its reconstitution of major matrix components. In support of our findings, Paul et al. previously reported that adenoviral vectors carrying the Sox 9 gene are able to increase the production of type II collagen in degenerated human disc cells. ${ }^{20}$ Unfortunately, adenoviral vectors can only mediate transient gene expression, and their in vivo application is limited due to the virus-induced immune response. As such, lentiviral vectors carrying Sox9, which mediate long-term transgene expression without severe immunogenic activity, offer greater advantages over adenoviruses.

Notably, our study demonstrated that a cocktail delivery of MMP3-shRNA and Sox9 lentiviruses dramatically blocked lumbar disc degeneration in rabbits. Even 24 weeks after combined injection, a time point when extremely severe disc degeneration was observed in the animals injected with PBS or an empty virus, MRI scans still identified intact disc tissues, with the highest signal intensity among all lentivirus-treated groups. Interestingly, Moon et al. reported that combination therapy of TGF $\beta 1$, IGF1 and BMP2 adenoviral vectors is better than single-gene therapy because multiple gene transduction enables enhanced biological responses, such as proteoglycan synthesis. ${ }^{21}$ Likewise, in our study, the combined gene transfer of lentiviral MMP3-shRNA and Sox9 vectors displayed greater capacity than the injection of LV-MMP3-shRNA or LV-Sox9 alone in the induction of collagen II expression and proteoglycan production, suggesting that combined gene delivery triggers a synergistic effect in ECM synthesis. The mechanism underlying this synergy seems likely to be mediated through the simultaneous inhibition of matrix degradation (via MMP3 knockdown) and enhancement of matrix induction (via Sox 9 overexpression), thereby leading to maximized matrix production. Notably, we observed that delivery of the 2 lentiviruses reduced MMP3 - or increased Sox9 - mRNA to a level comparable to that of LV-MMP3-shRNA or LV-Sox9 gene delivery alone. We assume that there may be an interaction between the 2 transgenes to further impact their expression, resulting in augmented Sox9 or greatly reduced MMP3 expression. Our assumption is partially supported by a recent study, in which Sox9 was able to inhibit the enzymatic activities of MMP2 and MMP13 through its binding with the MMP gene promoter. ${ }^{22}$

In general, our results demonstrate that lentivirus-mediated in vivo gene therapy targeting MMP3 or overexpressing Sox9 is efficient in delaying lumbar disc degeneration by restoring 2 major NP matrix components, collagen II and proteoglycan. Our results also suggest that gene therapy delivering MMP3-shRNA and Sox9 lentiviruses offers a promising approach for maximizing matrix reconstitution with a minimal dose of lentivirus mixture.

\section{ORCID iDs}

Zheng Zhao (1) https://orcid.org/0000-0002-6523-3425 Siyuan Li (1) https://orcid.org/0000-0003-4220-4493 Hui Huang (1) https://orcid.org/0000-0002-7600-2331 Jing Fang (i) https://orcid.org/0000-0002-3372-8650 Huawei Wei (1) https://orcid.org/0000-0001-7483-0902 Yongming Xi (1) https://orcid.org/0000-0002-0861-6147

\section{References}

1. Hodgkinson T, Shen B, Diwan A, Hoyland JA, Richardson SM. Therapeutic potential of growth differentiation factors in the treatment of degenerative disc diseases. JOR Spine. 2019;2(1):e1045.

2. Lyu FJ, Cheung KM, Zheng Z, Wang H, Sakai D, Leung VY. IVD progenitor cells: A new horizon for understanding disc homeostasis and repair. Nat Rev Rheumatol. 2019;15(2):102-112.

3. Gruber HE, Hoelscher GL, Ingram JA, Hanley EN Jr. Matrix metalloproteinase-26, a novel MMP, is constitutively expressed in the human intervertebral disc in vivo and in vitro. Exp Mol Pathol. 2012;92(1): 59-63.

4. Eser B, Eser O, Yuksel Y, et al. Effects of MMP-1 and MMP-3 gene polymorphisms on gene expression and protein level in lumbar disc herniation. Genet Mol Res. 2016;15(3). doi:10.4238/gmr.15038669

5. Lv FJ, Peng Y, Lim FL, et al. Matrix metalloproteinase 12 is an indicator of intervertebral disc degeneration co-expressed with fibrotic markers. Osteoarthr Cartil. 2016;24(10):1826-1836.

6. Zigouris A, Alexiou GA, Batistatou A, Voulgaris S, Kyritsis AP. The role of matrix metalloproteinase 9 in intervertebral disc degeneration. J Clin Neurosci. 2011;18(10):1424-1425.

7. Bachmeier BE, Nerlich A, Mittermaier N, et al. Matrix metalloproteinase expression levels suggest distinct enzyme roles during lumbar disc herniation and degeneration. Eur Spine J. 2009;18(11):1573-1586.

8. Lefebvre V, Angelozzi M, Haseeb A. SOX9 in cartilage development and disease. Curr Opin Cell Biol. 2019;61:39-47.

9. Sive JI, Baird P, Jeziorsk M, Watkins A, Hoyland JA, Freemont AJ. Expression of chondrocyte markers by cells of normal and degenerate intervertebral discs. Mol Pathol. 2002;55(2):91-97.

10. Gruber HE, Norton HJ, Ingram JA, Hanley EN Jr. The SOX9 transcription factor in the human disc: Decreased immunolocalization with age and disc degeneration. Spine (Phila Pa 1976). 2005;30(6):625-630.

11. Sobajima S, Kim JS, Gilbertson LG, Kang JD. Gene therapy for degenerative disc disease. Gene Ther. 2004;11(4):390-401.

12. Nishida K, Kang JD, Gilbertson LG, et al. Modulation of the biologic activity of the rabbit intervertebral disc by gene therapy: An in vivo study of adenovirus-mediated transfer of the human transforming growth factor beta 1 encoding gene. Spine (Phila Pa 1976). 1999; 24(23):2419-2425. 
13. Nishida K, Suzuki T, Kakutani K, et al. Gene therapy approach for disc degeneration and associated spinal disorders. Eur Spine J. 2008; 17(Suppl 4):459-466.

14. Ren S, Liu Y, Ma J, et al. Treatment of rabbit intervertebral disc degeneration with co-transfection by adeno-associated virus-mediated SOX9 and osteogenic protein-1 double genes in vivo. Int J Mol Med. 2013;32(5):1063-1068

15. Masuda K, Aota Y, Muehleman C, et al. A novel rabbit model of mild, reproducible disc degeneration by an anulus needle puncture: Correlation between the degree of disc injury and radiological and histological appearances of disc degeneration. Spine (Phila Pa 1976). 2005;30(1):5-14.

16. Kwon YJ. A minimally invasive rabbit model of progressive and reproducible disc degeneration confirmed by radiology, gene expression, and histology. J Korean Neurosurg Soc. 2013;53(6):323-330.

17. Lei T, Zhang Y, Zhou Q, et al. A novel approach for the annulus needle puncture model of intervertebral disc degeneration in rabbits. Am J Transl Res. 2017;9(3):900-909.
18. Vo NV, Hartman RA, Yurube T, Jacobs LJ, Sowa GA, Kang JD. Expression and regulation of metalloproteinases and their inhibitors in intervertebral disc aging and degeneration. Spine J. 2013;13(3):331-341.

19. Newell N, Carpanen D, Evans JH, Pearcy MJ, Masouros SD. Mechanical function of the nucleus pulposus of the intervertebral disc under high rates of loading. Spine (Phila Pa 1976). 2019;44(15):1035-1041.

20. Paul R, Haydon RC, Cheng $\mathrm{H}$, et al. Potential use of Sox9 gene therapy for intervertebral degenerative disc disease. Spine (Phila Pa 1976). 2003;28(8):755-763.

21. Moon SH, Nishida K, Gilbertson LG, et al. Biologic response of human intervertebral disc cells to gene therapy cocktail. Spine (Phila Pa 1976). 2008;33(17):1850-1855.

22. Luo $H$, Wang $C$, Liu $M$, et al. Inhibition of SOX9 promotes inflammatory and immune responses of dental pulp. J Endod. 2018;44(5): 792-799. 